\title{
A GEOMETRIC DRIFT INEQUALITY FOR A REFLECTED FRACTIONAL BROWNIAN MOTION PROCESS ON THE POSITIVE ORTHANT
}

\author{
CHIHOON LEE, ${ }^{*}$ Colorado State University
}

\begin{abstract}
We study a $d$-dimensional reflected fractional Brownian motion (RFBM) process on the positive orthant $S=\mathbb{R}_{+}^{d}$, with drift $r^{0} \in \mathbb{R}^{d}$ and Hurst parameter $H \in\left(\frac{1}{2}, 1\right)$. Under a natural stability condition on the drift vector $r^{0}$ and reflection directions, we establish a geometric drift towards a compact set for the 1-skeleton chain $\breve{Z}$ of the RFBM process $Z$; that is, there exist $\beta, b \in(0, \infty)$ and a compact set $C \subset S$ such that $\Delta V(x):=\mathrm{E}_{x}[V(\breve{Z}(1))]-V(x) \leq-\beta V(x)+b \mathbf{1}_{C}(x), x \in S$, for an exponentially growing Lyapunov function $V: S \rightarrow[1, \infty)$. For a wide class of Markov processes, such a drift inequality is known as a necessary and sufficient condition for exponential ergodicity. Indeed, similar drift inequalities have been established for reflected processes driven by standard Brownian motions, and our result can be viewed as their fractional Brownian motion counterpart. We also establish that the return times to the set $C$ itself are geometrically bounded. Motivation for this study is that RFBM appears as a limiting workload process for fluid queueing network models fed by a large number of heavy-tailed ON/OFF sources in heavy traffic.
\end{abstract}

Keywords: Reflected fractional Brownian motion; heavy traffic theory; geometric drift inequality; return time

2010 Mathematics Subject Classification: Primary 60G22

Secondary 90B18; 60G15; 60G18

\section{Introduction}

We consider a multidimensional reflected fractional Brownian motion (RFBM) process $\{Z(t): t \geq 0\}$ on the positive orthant $S:=\mathbb{R}_{+}^{d}$, with drift $r^{0} \in \mathbb{R}^{d}$ and Hurst parameter $H \in\left(\frac{1}{2}, 1\right)$. This model serves as an approximation for stochastic networks with a large number of heavy-tailed ON/OFF sources (see [4]), or with long-range dependent arrival and service time processes (see [14] and [17]), in the heavy traffic regime. This model was further studied in the subsequent papers [5] and [6], where the heavy traffic limit theorems for a multiclass multiserver fluid network and various asymptotic behaviors of the (weighted) maximum processes were studied, respectively. The logarithmic asymptotics of the tail probabilities of such reflected processes were obtained (cf. [16] and the references therein). In a recent paper [15], a uniform moment estimate on expected return times of the RFBM process to a compact set was established. While there is an ample body of literature for heavy traffic analysis based on short-range dependent (Markovian-like) models and light tails, the study on the long-time stability properties of the RFBM process is a largely unexplored area. This is mainly due to the lack of semimartingale and/or Markov properties of fractional Brownian motion (FBM) and,

Received 6 January 2011; revision received 18 April 2011.

* Postal address: Department of Statistics, Colorado State University, Fort Collins, CO 80523, USA.

Email address: chihoon@stat.colostate.edu 
hence, many techniques from the classical theory of stochastic calculus are inapplicable to its analysis.

In this work we establish a few basic stability properties of the RFBM process, mainly by proving the geometric drift towards a compact set $C \subset S$ for the discretely sampled RFBM process $\{\breve{Z}(n): n \geq 0\}$. More precisely, we show that $\{\breve{Z}(n):=Z(n), n=0,1, \ldots\}$ satisfies the following drift inequality. There exist $\beta, b \in(0, \infty)$ and a compact set $C \subset S$ such that

$$
\Delta V(x):=\mathrm{E}_{x}[V(\breve{Z}(1))]-V(x) \leq-\beta V(x)+b \mathbf{1}_{C}(x), \quad x \in S,
$$

with a suitable Lyapunov function $V: S \rightarrow[1, \infty)$ satisfying an exponential growth condition. Here (and throughout the paper), $\mathrm{E}_{x}$ denotes the expectation conditional on the process $\breve{Z}$ starting from $x \in S$ and $\mathbf{1}_{C}(\cdot)$ denotes the indicator function of a set $C$. This result (commonly referred to as a geometric drift condition (V4) in the literature-cf. Chapter 15 of [20]), combined with a petite set requirement for $C$, is reminiscent of a necessary and sufficient condition for the exponential ergodicity of a $\psi$-irreducible, aperiodic Markov process (see, e.g. [7], [19], and [20, pp. 393-394]). Similar results as in (1.1) were established for reflected processes driven by standard Brownian motions; indeed, the $V$-uniform ergodicity result of [3] (see also [11] for an ergodicity result) in view of Theorem 5.3 of [7] implies (1.1) for semimartingale reflecting Brownian motions. In this regard, the results in this paper can be viewed as a significant step towards further time-asymptotic analysis of RFBM with the aim of establishing similar ergodic properties for reflected processes driven by non-Markovian processes.

The organization of the paper is as follows. In Section 2 we carefully describe our model in Definition 2.1 and make a standard assumption on the reflection matrix (see assumption (HR) in Section 2), which is used in heavy traffic analysis for invoking a functional central limit theorem in [4] and [5]. In addition, similar to [13], we assume a natural stability condition (see condition (S) in Section 2) on the RFBM process. Our proof of the main result is based on exploiting basic properties, such as Lipschitz continuity and linear growth, of a hitting time to the origin function $T(\cdot)$ (see Lemma 3.1) on a family of certain deterministic dynamical systems obtained from the underlying RFBM process. This result, combined with an exponential moment estimate for the maximal increment of FBM with $H \in\left(\frac{1}{2}, 1\right)$ yields an estimate on the expected exponential hitting times to the origin in terms of the initial state (Lemma 3.3). In order to connect this result with the desired drift inequality (1.1), we make a suitable choice of $\delta \in(0, \infty)$ and show that $V(\breve{Z}(n)):=\mathrm{e}^{\delta T(\breve{Z}(n))}$ is bounded in mean on some compact set $C \subset S$, and also that it has a uniform negative drift off of the set $C$ (see Theorem 3.1).

As a consequence of the geometric drift inequality, we obtain the comparison result (Theorem 3.2) and then establish an exponential regularity result for the sampled process (see Theorem 3.3). Such results are used to conclude that the return times to the set $C$ itself are geometrically bounded. Lastly, we show in Theorem 3.4 that the drift inequality (1.1) can be extended with a larger compact set $D \subset S$ (in place of $C$ ), and, hence, the subsequent exponential regularity results hold on the set $D$ as well.

We use the following notation. The set of positive integers is denoted by $\mathbb{N}$, and we let $\mathbb{N}_{0}=\mathbb{N} \cup\{0\}$. The set of real numbers is denoted by $\mathbb{R}$ and the nonnegative real numbers by $\mathbb{R}_{+}$. For $a \in \mathbb{R}$, let $a^{+}=\max \{a, 0\}$. Let $\mathbb{R}^{d}$ be the $d$-dimensional Euclidean space, and, for $x \in \mathbb{R}^{d}$, the $L_{1}$-norm of $x$, i.e. $\sum_{i=1}^{d}\left|x_{i}\right|$, will be denoted by $|x|$. For a $(d \times d)$-dimensional matrix $A$, we will use the norm $\|A\|=\max _{1 \leq j \leq d} \sum_{i=1}^{d}\left|a_{i j}\right|$. For a given matrix $M$, denote by $M^{\top}$ its transpose and by $M_{i}$ the $i$ th row of $M$. Let $\mathcal{I}=\mathcal{I}_{d \times d}$ denote the identity matrix for some $d$. When it is clear from the context, we will omit the subscript. For a set $A \subset \mathbb{R}^{d}$, denote 
its interior and boundary by $A^{\circ}$ and $\partial A$, respectively. For sets $A, B \subset \mathbb{R}^{d}$, $\operatorname{dist}(A, B)$ will denote the distance between two sets, i.e. $\inf \{|x-y|: x \in A, y \in B\}$. Let $C(X, Y)$ denote the space of continuous functions from $X$ to $Y$, endowed with the topology of uniform convergence on compact intervals. Inequalities for vectors are interpreted componentwise.

\section{Model and assumptions}

We begin with the definitions of multidimensional FBM and RFBM. Let $d \in \mathbb{N}$. A stochastic process $B_{H}=\left\{B_{H}(t)=\left(B_{H}^{(1)}(t), \ldots, B_{H}^{(d)}(t)\right)^{\top}, t \geq 0\right\}$, defined on some filtered probability space $\left(\Omega, \mathcal{F},\left(\mathcal{F}_{t}\right)_{t \geq 0}, \mathrm{P}\right)$, is called a $d$-dimensional FBM of (Hurst) parameter $H \in(0,1)$, starting from $B_{H}(0) \in \mathbb{R}^{d}$, and associated matrix $\Lambda$, if it satisfies the following conditions. The process $B_{H}$ is a continuous Gaussian process with initial condition $B_{H}(0), \mathrm{P}$-almost surely (P-a.s.) and its covariance function is given by

$$
\operatorname{cov}\left(B_{H}(t), B_{H}(s)\right)=\mathrm{E}\left[\left(B_{H}(t)-B_{H}(0)\right)\left(B_{H}(s)-B_{H}(0)\right)^{\top}\right]=\Lambda_{H}(s, t) \Lambda
$$

for any $s, t \geq 0$, where $\Lambda$ is a $d \times d$ positive definite matrix and

$$
\Lambda_{H}(s, t):=\frac{1}{2}\left(t^{2 H}+s^{2 H}-|t-s|^{2 H}\right) .
$$

Without loss of generality, we will assume throughout that the diagonal entries of $\Lambda$ are all $1 \mathrm{~s}$. Also, it is assumed that $B_{H}$ is adapted to the filtration $\left(\mathcal{F}_{t}\right)_{t \geq 0}$. We will say that $B_{H}$ is a $d$-dimensional FBM with associated data $\left(B_{H}(0), H, \Lambda\right)$.

Fix some column vectors $r^{0}, r^{1}, \ldots, r^{d} \in \mathbb{R}^{d}$, and define the matrix $R:=\left[r^{1}, \ldots, r^{d}\right]_{d \times d}$. The quintuple $\left(B_{H}(0), H, \Lambda, r^{0}, R\right)$ will be called the data for an RFBM. The following definition is similar to that given in [4].

Definition 2.1. (RFBM.) For $x \in S$, an RFBM associated with the data $\left(x, H, \Lambda, r^{0}, R\right)$ is a continuous $d$-dimensional process $Z_{x}$, defined on some probability space $(\Omega, \mathcal{F}, \mathrm{P})$, such that

(i) $Z_{x}(t)=x+B_{H}(t)+r^{0} t+R Y(t) \in S$ for all $t \geq 0$, P-a.s.,

(ii) $B_{H}$ is a $d$-dimensional FBM with data $(0, H, \Lambda)$,

(iii) $Y$ is a $d$-dimensional process such that $Y_{i}(0)=0$ for $i=1, \ldots, d$, P-a.s. For each $i=1, \ldots, d, Y_{i}$ is continuous, nondecreasing, and can increase only when $Z(\cdot)$ is on the face $F^{i}:=\left\{x \in S: x_{i}=0\right\}$, i.e. $\int_{0}^{t} \mathbf{1}_{\left\{Z_{i}(s) \neq 0\right\}} \mathrm{d} Y_{i}(s)=0$ for all $t \geq 0$.

The set of directions of reflections on the boundary is defined as follows. For $y \in \partial S$,

$$
r(y):=\left\{\sum_{i=1}^{d} q_{i} r^{i}: \sum_{i=1}^{d} q_{i}=1, q_{i} \geq 0, \text { and } q_{i}>0 \text { only if } y_{i}=0\right\} .
$$

An RFBM, introduced in the above definition, behaves like an FBM in the interior of the orthant $S$ and it is confined to the orthant by an instantaneous 'reflection' (or, more accurately, 'regulation') at the boundary $\partial S$. For each $i$, the $i$ th column of the reflection matrix $R$ gives the direction of the reflection on the $i$ th face $F^{i}$. Specifically, if the boundary $F^{i}$ is hit, it is $Y_{i}$ that increases, the direction of displacement is given by $r^{i}$, the $i$ th column of $R$, and the magnitude of the displacement is the minimal amount required to keep $Z_{i}$ nonnegative. At an intersection of faces, the allowed directions of reflection are given by the convex combinations of the reflection directions associated with the faces meeting there (cf. [23]). 
A square matrix $R$ is called completely- $\$$ if, for every $k \times k$ principal submatrix $G$ of $R$, there is a $k$-dimensional vector $v_{G}$ such that $v_{G} \geq 0$ and $G v_{G}>0$. The completely- $\&$ condition on the reflection matrix $R$ ensures that, for every $y \in \partial S$, there exists a convex combination of vectors in $r(y)$ which points into $S^{\circ}$ from $y$. Also, the completely- $\&$ property is sufficient to ensure the existence of a pair $(Z, Y)$ satisfying Definition 2.1(i) and (iii) (cf. Theorem 2 of [2]). However, this property does not ensure the adaptness of the process $Y$ to a filtration to which $B_{H}$ is adapted. This problem is overcome under a stronger assumption on $R$, which we quote below (cf. Proposition 4.2 of [22] and Section 2 of [4]). We will impose throughout the following assumption on the reflection matrix $R$.

(HR) (i) The reflection matrix $R$ can be expressed as $\mathcal{I}+\Theta$, where $\Theta$ is a $(d \times d)$-dimensional matrix such that $|\Theta|$, that is, the matrix obtained from $\Theta$ by replacing all the entries in $\Theta$ by their absolute values, has spectral radius $r(|\Theta|)$ strictly less than 1 .

(ii) Moreover, the matrix $\Theta=\left(\theta_{i j}\right)$ satisfies $\theta_{i j} \leq 0$ and $\theta_{i i}=0$ for $1 \leq i, j \leq d$.

We now describe some of the important implications of assumption (HR). Under (HR), it can be shown (cf. [4] and [22]) that if $B_{H}$ is adapted to some filtration $\left\{\mathscr{F}_{t}: t \geq 0\right\}$ then $(Z, Y)$ is adapted to the filtration $\left\{g_{t}: t \geq 0\right\}$, with $g_{t}=\mathcal{F}_{t} \vee \mathcal{N}$, where $\mathcal{N}$ denotes the collection of P-null sets in $\mathcal{F}$. Henceforth, with an abuse of notation, we will assume that $(Z, Y)$ is adapted to the filtration $\left\{\mathcal{F}_{t}: t \geq 0\right\}$. We also note that part (i) of (HR) implies that the matrix $R$ is completely- $\&$ and invertible. Furthermore, part (i) of (HR) is a sufficient condition for strong pathwise uniqueness of a solution of the Skorokhod problem described below. Part (ii) of (HR), together with part (i), verifies the so-called Harrison-Reiman condition in [12]. As a consequence, (HR) will imply that a solution to the Skorokhod problem exists, and, moreover, the Skorokhod map is Lipschitz continuous in the sense of Proposition 2.1 below.

Definition 2.2. (Skorokhod problem.) Let $\psi \in C\left([0, \infty), \mathbb{R}^{d}\right)$ be given with $\psi(0) \in S$. Then, we say that $(\phi, \eta) \in C\left([0, \infty), \mathbb{R}^{d}\right) \times C\left([0, \infty), \mathbb{R}^{d}\right)$ solves the Skorokhod problem for $\psi$ with respect to $S$ and $R$ if and only if the following assertions hold:

(i) $\phi(t)=\psi(t)+R \eta(t) \in S$ for all $t \geq 0$,

(ii) for $1 \leq i \leq d$, (a) $\eta_{i}(0)=0$, (b) $\eta_{i}$ is nondecreasing, and (c) $\eta_{i}$ can increase only when $\phi$ is on the $i$ th face of $S$, that is, $\int_{0}^{\infty} \mathbf{1}_{\left\{\phi_{i}(s) \neq 0\right\}} \mathrm{d} \eta_{i}(s)=0$.

Let $C_{S}\left([0, \infty), \mathbb{R}^{d}\right):=\left\{\psi \in C\left([0, \infty), \mathbb{R}^{d}\right): \psi(0) \in S\right\}$. On the domain $E \subset C_{S}([0, \infty)$, $\mathbb{R}^{d}$ ), on which there is a unique solution to the Skorokhod problem, we define the Skorokhod map $\Gamma$ as

$$
\Gamma(\psi):=\phi,
$$

if $\left(\phi, R^{-1}[\phi-\psi]\right)$ is the unique solution of the Skorokhod problem posed by $\psi$. An equivalent form of RFBM in Definition 2.1 in terms of the Skorokhod map can now be written as

$$
Z=\Gamma\left(x+B_{H}+r^{0} \imath\right), \quad Z-\left(x+B_{H}+r^{0} \imath\right)=R Y,
$$

where $l:[0, \infty) \rightarrow[0, \infty)$ is the identity map, i.e. $l(t)=t$ for $t \in[0, \infty)$.

As a consequence of assumption (HR), we have the following proposition on the regularity of the Skorokhod map [12]. (Although Lipschitz continuity is not stated explicitly in [12], it follows easily from the method used to prove the existence of solutions and continuity of the Skorokhod map (map $\phi(\cdot)$ in [12]).) See also [8], [9], and [10] for more general sufficient conditions under which this regularity property holds. 
Proposition 2.1. The Skorokhod map is well defined on all of $C_{S}\left([0, \infty), \mathbb{R}^{d}\right)$, i.e. $E=$ $C_{S}\left([0, \infty), \mathbb{R}^{d}\right)$, and the Skorokhod map is Lipschitz continuous in the following sense. There exists a constant $L \in(0, \infty)$ such that, for all $\psi_{1}, \psi_{2} \in C_{S}\left([0, \infty), \mathbb{R}^{d}\right)$ and $t \geq 0$,

$$
\sup _{0 \leq s \leq t}\left|\Gamma\left(\psi_{1}\right)(s)-\Gamma\left(\psi_{2}\right)(s)\right|<L \sup _{0 \leq s \leq t}\left|\psi_{1}(s)-\psi_{2}(s)\right| .
$$

Next, we introduce the main stability condition on the drift vector $r^{0}$ and the matrix $R$ that will be assumed throughout this paper.

(S) There exists a constant $\theta>0$ such that $\sup _{1 \leq i \leq d}\left[R^{-1} r^{0}\right]_{i}<-\theta$.

Remark 2.1. For a model driven by standard Brownian motion (i.e. when $H=\frac{1}{2}$ ), condition ( $\mathrm{S}$ ) is known as a necessary and sufficient condition for the existence of a unique stationary distribution for the reflected process $Z$. We refer the reader to [13] and the references therein for the related asymptotic stability results of reflected Brownian motions (RBMs) on a positive orthant.

\section{Main results}

Let $Z_{x}$ be defined as in Definition 2.1 with the initial condition $Z_{x}(0)=x \in S$ and Hurst parameter $H \in\left(\frac{1}{2}, 1\right)$. Fix $x \in S$, and write the RFBM $Z_{x}$ in Definition 2.1 as

$$
Z_{x}(t)=\Gamma\left(x+r^{0} \imath+B_{H}(\cdot)\right)(t), \quad t \geq 0,
$$

where $\imath:[0, \infty) \rightarrow[0, \infty)$ is the identity map. Define the corresponding deterministic trajectory of $\left\{Z_{x}(t): t \geq 0\right\}$ by

$$
z_{x}(t):=\Gamma\left(x+r^{0} l\right)(t), \quad t \geq 0 .
$$

Next, defining $\mathcal{C}:=\left\{v \in \mathbb{R}^{d}: R^{-1} v \leq 0\right\}$, it can be seen from condition (S) that there exists a $\rho \in(0, \infty)$ satisfying

$$
\operatorname{dist}\left(r^{0}, \partial \mathcal{C}\right) \geq \frac{1}{\left\|R^{-1}\right\|} \inf \left\{\left|R^{-1} r^{0}-R^{-1} v\right|: R^{-1} v=0, v \in \mathbb{R}^{d}\right\} \geq \frac{1}{\left\|R^{-1}\right\|} \theta d=: \rho>0,
$$

where $\theta \in(0, \infty)$ is as in condition $(\mathrm{S})$. Thus,

$$
r^{0} \in \mathcal{C}_{\rho}:=\{v \in \mathcal{C}: \operatorname{dist}(v, \partial \mathcal{C}) \geq \rho\} .
$$

The cone $\mathcal{C}_{\rho}$ was considered in [1] to stipulate the permissible drift vector field and to characterize the positive recurrence of a class constrained diffusion process in some polyhedral domain. In this work we will treat $\rho \in(0, \infty)$ as one of the parameters, which can be chosen appropriately. (See the main result presented in Theorem 3.1 below.)

For $x_{0} \in S$, denote by $\mathcal{K}_{\rho}\left(x_{0}\right)$ the collection of all trajectories $\phi_{\rho}:[0, \infty) \rightarrow S$ of the form

$$
\phi_{\rho}(t)=\Gamma\left(x_{0}+\varpi l\right)(t), \quad t \geq 0,
$$

where $\varpi$ ranges over all $\mathcal{C}_{\rho}$. For a fixed $x_{0} \in S$, we now define the 'hitting time to the origin' function $T: S \rightarrow[0, \infty)$ as

$$
T\left(x_{0}\right):=\sup _{\phi_{\rho} \in \mathcal{K}_{\rho}\left(x_{0}\right)} \inf \left\{t \in[0, \infty): \phi_{\rho}(t)=0\right\} .
$$

We refer the reader to Section 3 of [1] for the various stability properties of the deterministic 
trajectories defined in (3.2). The following result from [1] (see Lemma 3.1 and its proof therein) will be crucial in our analysis.

Lemma 3.1. ([1].) The function $T: S \rightarrow[0, \infty)$ defined in (3.3) satisfies the following properties.

(i) For some $c_{1} \in(0, \infty),|T(x)-T(y)| \leq c_{1}|x-y|$ for all $x, y \in S$.

(ii) For some $c_{2}, c_{3} \in(0, \infty), c_{2}|x| \leq T(x) \leq c_{3}|x|$ for all $x \in S$. Thus, in particular, for all $M \in(0, \infty)$, the set $\{x \in S: T(x) \leq M\}$ is compact.

(iii) Fix $x \in S$, and let $z \in \mathcal{K}_{\rho}(x)$. Then, for all $t>0, T\left(z_{x}(t)\right) \leq(T(x)-t)^{+}$.

Here the positive constants $c_{i}(i=1,2,3)$ depend only on $L \in(0, \infty)$ and $\rho \in(0, \infty)$, given in Proposition 2.1 and (3.1), respectively. In particular, $c_{1}=4 L^{3} \rho^{-1}$.

Next we present an exponential moment estimate for the maximal increment of the FBM $B_{H}$ with $H \in\left(\frac{1}{2}, 1\right)$, which may be of independent interest.

Lemma 3.2. Let $h \in(0, \infty)$ be a constant. For $n \in \mathbb{N}$, let $v_{n}$ be defined as

$$
v_{n}:=\sup \left\{\left|B_{H}(s)-B_{H}((n-1) h)\right|:(n-1) h \leq s \leq n h\right\},
$$

where $B_{H}(\cdot)$ is a d-dimensional FBM with data $(0, H, \Lambda)$ and Hurst parameter $H \in\left(\frac{1}{2}, 1\right)$. Then, for any $\gamma \in(0, \infty)$ and $n \in \mathbb{N}$,

$$
\mathrm{E}\left[\mathrm{e}^{\gamma v_{n}}\right] \leq 4 d \mathrm{e}^{\gamma^{2} d^{2} h^{2 H} / 2}
$$

where $d \geq 1$ is the dimension of the FBM $B_{H}$.

Proof. We begin by recalling that, for a positive random variable $v_{n}$,

$$
\mathrm{E}\left[\mathrm{e}^{\gamma v_{n}}\right]=1+\int_{0}^{\infty} \gamma \mathrm{e}^{\gamma y} \mathrm{P}\left[v_{n}>y\right] \mathrm{d} y .
$$

From the stationary increment and self-similar properties of FBM (cf. Theorem 3.3 of [18]), it follows that

$$
\begin{aligned}
v_{n} & \stackrel{\mathrm{D}}{=} \sup \left\{\left|B_{H}(s)-B_{H}(0)\right|: 0 \leq s \leq h\right\} \\
& \stackrel{\text { a.s. }}{=} \sup \left\{\left|B_{H}(s)\right|: 0 \leq s \leq h\right\} \\
& \stackrel{\mathrm{D}}{=} h^{H} \sup \left\{\left|B_{H}(s)\right|: 0 \leq s \leq 1\right\},
\end{aligned}
$$

where $\stackrel{\text { ㅁ․ }}{=}$ ' denotes equality in distribution and $\stackrel{\text { a.s., }}{=}$ follows from the assumption that $B_{H}(0)=0$, P-a.s. (Recall part (ii) of Definition 2.1.)

We therefore obtain

$$
\begin{aligned}
\mathrm{P}\left[v_{n}>y\right] & =\mathrm{P}\left[\sup \left\{\left|B_{H}(s)\right|: 0 \leq s \leq 1\right\}>h^{-H} y\right] \\
& \leq \mathrm{P}\left[\sum_{i=1}^{d} \sup \left\{\left|B_{H}^{(i)}(s)\right|: 0 \leq s \leq 1\right\}>h^{-H} y\right] \\
& \leq d \mathrm{P}\left[\sup \left\{\left|B_{H}^{(1)}(s)\right|: 0 \leq s \leq 1\right\}>\frac{h^{-H}}{d} y\right] \\
& \leq 2 d \mathrm{P}\left[\sup \left\{B_{H}^{(1)}(s): 0 \leq s \leq 1\right\}>\frac{h^{-H}}{d} y\right],
\end{aligned}
$$

where the last inequality follows from the symmetry property of FBM. 
For $H \in\left(\frac{1}{2}, 1\right)$, it is known from Statement 4.2 (part 2) of [21] that

$$
\mathrm{P}\left[\sup \left\{B_{H}^{(1)}(s): 0 \leq s \leq 1\right\}>\lambda\right] \leq \sqrt{\frac{2}{\pi}} \int_{\lambda}^{\infty} \mathrm{e}^{-x^{2} / 2} \mathrm{~d} x
$$

for any $\lambda \geq 0$. More precisely, (3.6) follows from setting $f=1$ and $r=1$ in Statement 4.2 of [21]. For a more direct presentation of this result, we refer the reader to the penultimate displayed inequality on page 1067 of [21]. (We note that since the definition of FBM in [21] is slightly different from ours, we should set $\alpha=2 H-1$, and the function $q_{f}(s, t)$ in Statement 4.2 should be corrected by multiplying a missing constant factor $C \alpha(\alpha+1)=H(2 H-1)$; see also the first displayed equality on page 1067.) Applying the estimate in (3.6) to (3.4) and (3.5), we obtain

$$
\begin{aligned}
\mathrm{E}\left[\mathrm{e}^{\gamma \nu_{n}}\right] & \leq 1+2 d \sqrt{\frac{2}{\pi}} \int_{0}^{\infty} \gamma \mathrm{e}^{\gamma y}\left[\int_{y /\left(d h^{H}\right)}^{\infty} \mathrm{e}^{-x^{2} / 2} \mathrm{~d} x\right] \mathrm{d} y \\
& =1+2 d \sqrt{\frac{2}{\pi}} \int_{0}^{\infty} \mathrm{e}^{-x^{2} / 2}\left[\int_{0}^{d h^{H} x} \gamma \mathrm{e}^{\gamma y} \mathrm{~d} y\right] \mathrm{d} x \\
& =1+2 d \sqrt{\frac{2}{\pi}} \int_{0}^{\infty} \mathrm{e}^{-x^{2} / 2}\left[\mathrm{e}^{\gamma d h^{H} x}-1\right] \mathrm{d} x .
\end{aligned}
$$

By recalling $\int_{0}^{\infty} \mathrm{e}^{-x^{2} / 2} \mathrm{~d} x=\sqrt{2 \pi} / 2$ and completing the square in the exponent, it can be shown that the last expression is bounded above by

$$
2 d \sqrt{\frac{2}{\pi}} \mathrm{e}^{\left(\gamma d h^{H}\right)^{2} / 2} \int_{0}^{\infty} \mathrm{e}^{-\left(x-\gamma d h^{H}\right)^{2} / 2} \mathrm{~d} x=4 d \mathrm{e}^{\left(\gamma d h^{H}\right)^{2} / 2}\left(1-\Phi\left(-\gamma d h^{H}\right)\right),
$$

where $\Phi(\cdot)$ denotes the cumulative distribution function of the standard normal random variable. Noting that $\Phi(\cdot) \in[0,1]$, we obtain the required bound.

Next, we define a '1-skeleton' process $\breve{Z}$ and an associated filtration. Recall that an RFBM process $\{Z(t): t \geq 0\}$ is defined on the filtered probability space $\left(\Omega, \mathcal{F},\left(\mathcal{F}_{t}\right)_{t \geq 0}, \mathrm{P}\right)$. For $k \geq 0$, we let

$$
\breve{Z}(k):=Z(k), \quad \breve{\mathcal{F}}_{k}:=\mathscr{F}_{k} .
$$

Combining Lemmas 3.1 and 3.2 yields the following result.

Lemma 3.3. For $\delta \in(0, \infty)$, there is a constant $c_{4} \in(0, \infty)$ such that

$$
\mathrm{E}_{x} \mathrm{e}^{\delta[T(\breve{Z}(1))]} \leq 4 d \mathrm{e}^{\delta[T(x)-1]} \mathrm{e}^{c_{4} \delta^{2}}, \quad t \geq 0,
$$

for all $x \in S_{1}:=\{x \in S: T(x)>1\}$.

Proof. Note that, for all $x \in S$,

$$
T\left(Z_{x}(1)\right)-T\left(z_{x}(1)\right) \leq c_{1}\left|Z_{x}(1)-z_{x}(1)\right| \leq c_{1} L \sup _{0 \leq s \leq 1}\left|B_{H}(s)\right|,
$$

where the first inequality follows from the Lipschitz property of $T$ (see Lemma 3.1(i)), and the second inequality follows from Proposition 2.1. Then, from Lemma 3.1(iii) we have, $\mathrm{P}_{x}$-a.s.,

$$
T\left(Z_{x}(1)\right) \leq(T(x)-1)^{+}+c_{1} L \sup _{0 \leq s \leq 1}\left|B_{H}(s)\right| \quad \text { for all } x \in S .
$$


Then, for $\delta \in(0, \infty)$ and $x \in S_{1}$, we obtain

$$
\mathrm{e}^{\delta\left[T\left(\breve{Z}_{x}(1)\right)\right]} \leq \mathrm{e}^{\delta[T(x)-1]} \mathrm{e}^{\delta c_{1} L \sup _{0 \leq s \leq 1}\left|B_{H}(s)\right|} \quad \mathrm{P}_{x} \text {-a.s. }
$$

Taking the expectation of both sides and using Lemma 3.2 (with $n=1, \gamma=\delta c_{1} L$, and $h=1$ ) yields (3.7), setting $c_{4}:=\frac{1}{2} c_{1}^{2} L^{2} d^{2}$ (which is proportional to $\rho^{-2}$ in view of Lemma 3.1).

Hereafter, we impose an assumption on the parameter $\rho \in(0, \infty)$ in $(3.1)$ so that the constant $c_{4} \in(0, \infty)$ in Lemma 3.3 can be small enough in the following analysis. Recall that $c_{1}=4 L^{3} \rho^{-1}$ and $c_{4}=\frac{1}{2} c_{1}^{2} L^{2} d^{2}$ from Lemma 3.1 and Lemma 3.3, respectively. In what follows, we choose $\rho \in(0, \infty)$ appropriately so that $c_{4}<(4 \log 4 d)^{-1}$. This amounts to assuming that $\rho \in(\kappa, \infty)$, where $\kappa:=4 d L^{4} \sqrt{2 \log 4 d}$. We now present the main result on the geometric drift inequality for the 1-skeleton RFBM process.

Theorem 3.1. (Geometric drift towards $C$.) For $x \in S$, let the RFBM process $\left\{Z_{x}(t)\right\}_{t \geq 0}$ be given as in Definition 2.1 and assume that $\rho \in(\kappa, \infty)$. Then, the 1-skeleton process $\left\{\breve{Z}_{x}(n):=Z_{x}(n)\right\}_{n \in \mathbb{N}_{0}}$ satisfies the following drift inequality. There exist $\delta, \beta, b \in(0, \infty)$ and a compact set $C \subset S$ such that

$$
\Delta V(x) \leq-\beta V(x)+b \mathbf{1}_{C}(x), \quad x \in S,
$$

with $V(x):=\mathrm{e}^{\delta T(x)}$.

Proof. From Lemma 3.3, for $\delta \in(0, \infty)$ and $x \in S_{1}:=\{x \in S: T(x)>1\}$,

$$
V(x)^{-1}\left[\mathrm{E}_{x} \mathrm{e}^{\delta[T(\breve{Z}(1))]}\right] \leq 4 d \mathrm{e}^{-\delta} \mathrm{e}^{c_{4} \delta^{2}},
$$

where $c_{4} \in(0, \infty)$ is a constant (independent of $\delta$ and $x$, and proportional to $\rho^{-2}$ ) as in (3.7). Now choose $\delta:=1 / 2 c_{4} \in(0, \infty)$ so that $c_{4} \delta^{2}-\delta+\log 4 d<0$ and, therefore, $\mathrm{e}^{c_{4} \delta^{2}-\delta+\log 4 d}:=$ $1-\beta<1$. Then we have

$$
\mathrm{E}_{x} V(\breve{Z}(1)) \leq(1-\beta) V(x) \text { for all } x \in S_{1} .
$$

Also, for $x \in C:=S \backslash S_{1}$, we have, from inequality (3.8),

$$
\mathrm{E}_{x} V(\breve{Z}(1)) \leq 4 d \mathrm{e}^{c_{4} \delta^{2}}=: b .
$$

Combining (3.10) and (3.11) we obtain

$$
\mathrm{E}_{x} V(\breve{Z}(1)) \leq(1-\beta) V(x)+b \mathbf{1}_{C}(x) \text { for all } x \in S,
$$

and (3.9) follows.

Remark 3.1. In view of Lemma 3.1(ii), the set $C=\{x \in S: T(x) \leq 1\}$ is compact and the function $V: S \rightarrow[1, \infty)$ is shown to be unbounded and to satisfy an exponential growth condition. We remark that similar drift inequalities as in (3.9) were established for a wide class of Markov processes [3], [7], [20, Chapter 16]. The geometric drift result in the form of (3.9) is known as the necessary and sufficient condition for the $V$-uniform ergodicity of underlying Markov processes. Indeed, the proof that geometric ergodicity of the 1-skeleton process (which follows from (3.9) for Markov processes) implies $V$-uniform ergodicity of the semigroup is a simple consequence of the submultiplicative property of the $V$-norm operator (cf. Theorems 5.2-5.3 of [7]). In this respect, the geometric drift result in Theorem 3.1 sheds some light on the time-asymptotic analysis of an RFBM process, anticipating similar long-time properties of reflected processes driven by standard Brownian motions. 
Remark 3.2. The geometric drift condition (3.9) can be written in the following simpler contractive form:

$$
\mathrm{E}_{x} V(\breve{Z}(1)) \leq(1-\beta) V(x)+b \quad \text { for all } x \in S .
$$

Here $1-\beta<1$ and $b$ are as in (3.9). Indeed, if (3.9) holds then (3.13) immediately follows. Conversely, if (3.13) holds then it can be shown that

$$
\Delta V(x) \leq-\bar{\beta} V(x)+b \mathbf{1}_{\bar{C}}(x), \quad x \in S,
$$

where $\bar{\beta}:=\beta / 2$ and $\bar{C}:=\{x \in S: V(x) \leq 2 b / \beta\}$.

As a consequence of the geometric drift towards a compact set $C$ in Theorem 3.1, we obtain the following comparison theorem, which will be useful for proving that the return times to the set $C$ itself are geometrically bounded. For $m \geq 1$ and a set $B \subset S$, define the first entrance time to the set $B$ by

$$
\breve{\tau}_{B}:=\inf \{m \geq 1: \breve{Z}(m) \in B\} .
$$

Theorem 3.2. For any $r \in\left(1,(1-\beta)^{-1}\right)$, there exists $\epsilon>0$ such that, for any first entrance time $\breve{\tau}_{B}$,

$$
\mathrm{E}_{x}\left[\sum_{k=0}^{\breve{\tau}_{B}-1} V(\breve{Z}(k)) r^{k}\right] \leq \epsilon^{-1} r^{-1} V(x)+\epsilon^{-1} b \mathrm{E}_{x}\left[\sum_{k=0}^{\breve{\tau}_{B}-1} \mathbf{1}_{C}(\breve{Z}(k)) r^{k}\right],
$$

where the constants $\beta, b \in(0, \infty)$ and the set $C \subset S$ are as in Theorem 3.1.

Proof. The proof is adapted from Theorem 15.2.5 of [20]. We begin by observing that, for any $r \in\left(1,(1-\beta)^{-1}\right)$, we can choose $\epsilon \in(0, \beta)$ to be the solution to $r=1 /(1-\beta+\epsilon)$. Then, from (3.12) we have the bound

$$
\mathrm{E}_{x} V(\breve{Z}(1)) \leq r^{-1} V(x)-\epsilon V(x)+b \mathbf{1}_{C}(x) \text { for all } x \in S .
$$

It follows that

$$
\begin{aligned}
\mathrm{E}\left[V(\breve{Z}(k+1)) r^{k+1} \mid \breve{\mathcal{F}}_{k}\right] & \leq r^{k+1}\left\{r^{-1} V(\breve{Z}(k))-\epsilon V(\breve{Z}(k))+b \mathbf{1}_{C}(\breve{Z}(k))\right\} \\
& =V(\breve{Z}(k)) r^{k}-\epsilon V(\breve{Z}(k)) r^{k+1}+r^{k+1} b \mathbf{1}_{C}(\breve{Z}(k)) .
\end{aligned}
$$

For $n \geq 1$ and $r \in(1, \infty)$, define

$$
\tau_{B}^{n}:=\min \left\{n, \breve{\tau}_{B}, \inf \left\{k \geq 0: V(\breve{Z}(k)) r^{k} \geq n\right\}\right\} .
$$

The random time $\tau_{B}^{n}$ is a stopping time with respect to the filtration $\left\{\breve{\mathcal{F}}_{k}\right\}$, since it is the minimum of stopping times with respect to the same filtration. Note that, since $\left\{\tau_{B}^{n} \geq i\right\} \in \breve{\mathcal{F}}_{i-1}$, we have

$$
\mathrm{E}_{x}\left[V\left(\breve{Z}\left(\tau_{B}^{n}\right)\right) r^{\tau_{B}^{n}}\right]=\mathrm{E}_{x}[V(\breve{Z}(0))]+\mathrm{E}_{x}\left[\sum_{k=0}^{\tau_{B}^{n}-1}\left(\mathrm{E}_{x}\left[V(\breve{Z}(k+1)) r^{k+1} \mid \breve{\mathcal{F}}_{k}\right]-V(\breve{Z}(k)) r^{k}\right)\right] .
$$

Fix $N>0$. From (3.15) and (3.16), we see that

$$
0 \leq \mathrm{E}_{x}\left[V\left(\breve{Z}\left(\tau_{B}^{n}\right)\right) r^{\tau_{B}^{n}}\right] \leq V(x)+\mathrm{E}_{x}\left[\sum_{k=0}^{\tau_{B}^{n}-1}\left(r^{k+1} b \mathbf{1}_{C}(\breve{Z}(k))-\epsilon V(\breve{Z}(k)) r^{k+1} \wedge N\right)\right] .
$$


Hence, by adding a finite term to each side of (3.17), we get

$$
\begin{aligned}
\mathrm{E}_{x}\left[\sum_{k=0}^{\tau_{B}^{n}-1}\left(\epsilon V(\breve{Z}(k)) r^{k+1} \wedge N\right)\right] & \leq V(x)+\mathrm{E}_{x}\left[\sum_{k=0}^{\tau_{B}^{n}-1} r^{k+1} b \mathbf{1}_{C}(\breve{Z}(k))\right] \\
& \leq V(x)+\mathrm{E}_{x}\left[\sum_{k=0}^{\breve{\tau}_{B}-1} r^{k+1} b \mathbf{1}_{C}(\breve{Z}(k))\right] .
\end{aligned}
$$

Letting $n \rightarrow \infty$ and then $N \rightarrow \infty$ gives

$$
\mathrm{E}_{x}\left[\sum_{k=0}^{\breve{\tau}_{B}-1}\left(\epsilon V(\breve{Z}(k)) r^{k+1}\right)\right] \leq V(x)+\mathrm{E}_{x}\left[\sum_{k=0}^{\breve{\tau}_{B}-1} r^{k+1} b \mathbf{1}_{C}(\breve{Z}(k))\right]
$$

by the monotone convergence theorem. Finally, multiplying both sides of (3.18) by $\epsilon^{-1} r^{-1} \epsilon$ $(0, \infty)$ yields $(3.14)$.

As an immediate consequence of Theorem 3.2, we obtain the following result.

Theorem 3.3. There exists $r>1$ such that

$$
\sup _{x \in C} \mathrm{E}_{x}\left[\sum_{k=0}^{\breve{\tau}_{C}-1} V(\breve{Z}(k)) r^{k}\right]<\infty,
$$

where the set $C \subset S$ is as in Theorem 3.1. Hence, in particular, we have

$$
\sup _{x \in C} \mathrm{E}_{x}\left[r^{\breve{\tau} C}\right]<\infty .
$$

Remark 3.3. A set $C$ satisfying (3.19) or (3.20) is respectively known as a $V$-Kendall set or a Kendall set in the Markov processes literature (cf. Chapter 15 of [20]).

Proof of Theorem 3.3. By choosing $B=C$ in Theorem 3.2, we have

$$
V(x) \leq \mathrm{E}_{x}\left[\sum_{k=0}^{\breve{t}_{C}-1} V(\breve{Z}(k)) r^{k}\right] \leq \epsilon^{-1} r^{-1} V(x)+\epsilon^{-1} b \mathbf{1}_{C}(x) .
$$

Therefore, we obtain

$$
\sup _{x \in C} \mathrm{E}_{x}\left[\sum_{k=0}^{\breve{\tau}_{C}-1} V(\breve{Z}(k)) r^{k}\right] \leq \epsilon^{-1} r^{-1} \sup _{x \in C} V(x)+\epsilon^{-1} b \leq \epsilon^{-1} r^{-1} \mathrm{e}^{\delta}+\epsilon^{-1} b<\infty .
$$

Result (3.20) then straightforwardly follows by noting that $V(\breve{Z}(k)) \geq 1$ for all $k \geq 0$.

Next we show that Theorems 3.1, 3.2, and 3.3 hold with the sublevel sets of $V$, which can be chosen larger than the set $C=\{x \in S: T(x) \leq 1\}$. We refer the reader to Theorem 15.2.6 of [20] for similar results for a class of $\psi$-irreducible Markov processes.

Theorem 3.4. Let $\delta, \beta, b \in(0, \infty)$ and $C \subset S$ be as in Theorem 3.1. Then, there exist $\beta^{\prime}, c \in(0, \infty)$ and a compact set $D \supset C$ in $S$ such that

$$
\Delta V(x) \leq-\beta^{\prime} V(x)+c \mathbf{1}_{D}(x), \quad x \in S,
$$

and, therefore, Theorems 3.2-3.3 hold with $D$ in place of $C$. 
Proof. Fix $\ell^{-1} \in(1-\beta, 1)$, choose $M \in(0, \infty)$ sufficiently large so that

$$
\mathrm{e}^{\delta} \leq \frac{M+b}{\ell^{-1}-(1-\beta)}
$$

and define

$$
D:=\left\{x \in S: V(x) \leq \frac{M+b}{\ell^{-1}-(1-\beta)}\right\} .
$$

Then, it follows that $C \subset D$ and, moreover, the set $D$ is compact in view of Lemma 3.1(ii). Using Theorem 3.1, we have

$$
\begin{aligned}
\mathrm{E}_{x} V(\breve{Z}(1)) & \leq \ell^{-1} V(x)-\left(\ell^{-1}-(1-\beta)\right) V(x)+b \mathbf{1}_{C}(x) \\
& \leq \ell^{-1} V(x)-M \leq \ell^{-1} V(x) \text { for all } x \in D^{\mathrm{c}},
\end{aligned}
$$

from the definition of the set $D$. Also, it follows that

$$
\mathrm{E}_{x} V(\breve{Z}(1)) \leq(1-\beta) V(x)+b \leq c \quad \text { for all } x \in D,
$$

for some $c \in(0, \infty)$, since $V$ is bounded on $D$ by construction. Thus, we have shown that

$$
\mathrm{E}_{x} V(\breve{Z}(1)) \leq \ell^{-1} V(x)+c \mathbf{1}_{D}(x) \text { for all } x \in S,
$$

and, hence, (3.21) follows with $\beta^{\prime}:=1-\ell^{-1} \in(0, \infty)$. Hence, using Theorem 3.2 (replacing the sets $B$ and $C$ by the set $D)$, there exist $s \in(1, \infty)$ and $\varepsilon \in(0, \infty)$ such that

$$
\mathrm{E}_{x}\left[\sum_{k=0}^{\breve{\tau}_{D}-1} V(\breve{Z}(k)) s^{k}\right] \leq \varepsilon^{-1} s^{-1} V(x)+\varepsilon^{-1} c \mathbf{1}_{D}(x) .
$$

Since $V$ is bounded on $D$, this leads to

$$
\sup _{x \in D} \mathrm{E}_{x}\left[\sum_{k=0}^{\breve{t}_{D}-1} V(\breve{Z}(k)) s^{k}\right]<\infty,
$$

and, hence, $\sup _{x \in D} \mathrm{E}_{x}\left[s^{\breve{\tau}_{D}}\right]<\infty$.

\section{Acknowledgements}

This work was partially supported by the Simons Foundation, under grant number 209658. The author would like to thank the anonymous referee for carefully examining the paper and providing valuable comments.

\section{References}

[1] Atar, R., Budhiraja, A. And Dupuis, P. (2001). On positive recurrence of constrained diffusion processes. Ann. Prob. 29, 979-1000.

[2] Bernard, A. and el Kharroubi, A. (1991). Régulations déterministes et stochastiques dans le premier "orthant" de $\mathbf{R}^{n}$. Stoch. Stoch. Reports 34, 149-167.

[3] Budhiraja, A. AND LeE, C. (2007). Long time asymptotics for constrained diffusions in polyhedral domains. Stoch. Process. Appl. 117, 1014-1036.

[4] Delgado, R. (2007). A reflected fBm limit for fluid models with ON/OFF sources under heavy traffic. Stoch. Process. Appl. 117, 188-201. 
[5] Delgado, R. (2008). State space collapse for asymptotically critical multi-class fluid networks. Queueing Systems 59, 157-184.

[6] Delgado, R. (2010). On the reflected fractional Brownian motion process on the positive orthant: asymptotics for a maximum with application to queueing networks. Stoch. Models 26, 272-294.

[7] Down, D., Meyn, S. P. And Tweedie, R. L. (1995). Exponential and uniform ergodicity of Markov processes. Ann. Prob. 23, 1671-1691.

[8] Dupuis, P. AND IsHII, H. (1991). On Lipschitz continuity of the solution mapping to the Skorokhod problem, with applications. Stoch. Stoch. Reports 35, 31-62.

[9] Dupuis, P. And Ramanan, K. (1999). Convex duality and the Skorokhod problem. I. Prob. Theory Relat. Fields 115, 153-195.

[10] Dupuis, P. and Ramanan, K. (1999). Convex duality and the Skorokhod problem. II. Prob. Theory Relat. Fields 115, 197-236.

[11] Dupuis, P. and Williams, R. J. (1994). Lyapunov functions for semimartingale reflecting Brownian motions. Ann. Prob. 22, 680-702.

[12] Harrison, J. M. and Reiman, M. I. (1981). Reflected Brownian motion on an orthant. Ann. Prob. 9, $302-308$.

[13] Harrison, J. M. AND Williams, R. J. (1987). Brownian models of open queueing networks with homogeneous customer populations. Stochastics 22, 77-115.

[14] Konstantopoulos, T. and Lin, S.-J. (1996). Fractional Brownian approximations of queueing networks. In Stochastic networks (New York, 1995; Lecture Notes Statist. 117), Springer, New York, pp. 257-273.

[15] LEE, C. (2011). On the return time for a reflected fractional Brownian motion process on the positive orthant. J. Appl. Prob. 48, 145-153.

[16] MAJEwski, K. (2003). Large deviations for multidimensional reflected fractional Brownian motion. Stoch. Stoch. Reports 75, 233-257.

[17] Majewski, K. (2005). Fractional Brownian heavy traffic approximations of multiclass feedforward queueing networks. Queueing Systems 50, 199-230.

[18] Mandelbrot, B. B. And Van Ness, J. W. (1968). Fractional Brownian motions, fractional noises and applications. SIAM Rev. 10, 422-437.

[19] Meyn, S. P. And Tweedie, R. L. (1993). Stability of Markovian processes. III. Foster-Lyapunov criteria for continous-time processes. Adv. Appl. Prob. 25, 518-548.

[20] Meyn, S. P. And Tweedie, R. L. (2009). Markov Chains and Stochastic Stability, 2nd edn. Cambridge University Press.

[21] Ruzmaikina, A. A. (2000). Stieltjes integrals of Hölder continuous functions with applications to fractional Brownian motion. J. Statist. Phys. 100, 1049-1069.

[22] Williams, R. J. (1998). An invariance principle for semimartingale reflecting Brownian motions in an orthant. Queueing Systems 30, 5-25.

[23] Williams, R. J. (1998). Reflecting diffusions and queueing networks. In Proceedings of the International Congress of Mathematicians (Berlin, 1998), Vol. III, pp. 321-330. 